\title{
Molecular Genetic Analysis of Multi-drug Resistance in Indian Isolates of Mycobacterium tuberculosis
}

\author{
Noman Siddiqi//+, Md. Shamim/ ${ }^{++}$, NK Jain*, Ashok Rattan**, Amol Amin, \\ VM Katoch ${ }^{* * *}$, SK Sharma****, Seyed E Hasnain $/{ }^{+}$
}

National Institute of Immunology, New Delhi, 110067, India *New Delhi T.B. Centre, New Delhi, India **Department of Microbiology, A.I.I.M.S., New Delhi, India ***Central Jalma Institute of Leprosy, Agra, India ****Department of Medicine, A.I.I.M.S., New Delhi, India

A total of 116 isolates from patients attending the out-patient department at the All India Institute of Medical Sciences, New Delhi and the New Delhi Tuberculosis Centre, New Delhi, India were collected. They were analyzed for resistance to drugs prescribed in the treatment for tuberculosis. The drug resistance was initially determined by microbiological techniques. The Bactec 460TB system was employed to determine the type and level of resistance in each isolate. The isolates were further characterized at molecular level. The multi-drug loci corresponding to $\mathrm{rpo} \mathrm{b}$, gyr A, kat $\mathrm{G}$ were studied for mutation(s) by the polymerase chain reaction-single strand conformational polymorphism (PCR-SSCP) technique. The SSCP positive samples were sequenced to characterize the mutations in $\mathrm{rpo} \mathrm{b}$, and gyr A loci. While previously reported mutations in the gyr $\mathrm{A}$ and $\mathrm{rpo} \mathrm{b}$ loci were found to be present, several novel mutations were also scored in the rpo b locus.

Interestingly, analysis of the gyr A locus showed the presence of point mutation(s) that could not be detected by PCR-SSCP. Furthermore, rifampicin resistance was found to be an important marker for checking multi-drug resistance $(M D R)$ in clinical isolates of Mycobacterium tuberculosis. This is the first report on molecular genetic analysis of MDR tuberculosis from India, and highlights the increasing incidence of MDR in the Indian isolates of M. tuberculosis.

Key words: clinical isolates - gyr A gene - multi-drug resistance - Mycobacterium tuberculosis - rpo b gene polymerase chain reaction-single strand conformational polymorphism

Until recently, the common belief held that tuberculosis (TB) no longer posed a major threat to public health, at least in developed countries. However due to various reasons there is an increasing incidence of TB leading to high morbidity and mortality rates (Bloom \& Murray 1992). Furthermore, the association of TB with the AIDS pandemic leading to increase in fatality rates and emergence of multi-drug resistant (MDR) strains of Mycobacterium tuberculosis is a cause of grave concern worldwide (Iseman 1994).

Resistance to rifampicin, isoniazid and fluoroquinolones have been well studied and characterized at the molecular level (Honore \& Cole

\footnotetext{
This project was supported by a grant from the Department of Biotechnology, Ministry of Science and Technology, India.

${ }^{+}$Corresponding author. Fax: +9111-616.2125. E-mail: ehtesham@nii.ernet.in

${ }^{++}$Both authors contributed equally.

Received 15 June 1998

Accepted 30 July 1998
}

1993, Heym et al. 1993, Miller 1994, Takiff et al. 1994). Rifampicin resistance arises due to mutations in rpo b gene encoding the DNA-dependent RNA polymerase. The primary target of rifampicin is the b-subunit of RNA polymerase. The association of the RNA polymerase $\mathrm{b}$ (rpo b) subunit gene with resistance to rifampicin has been documented previously and subsequent reports from various groups have confirmed this association in clinical isolates of $M$. tuberculosis (Kapur et al. 1994, Williams et al. 1994, Musser 1995, Hasnain et al. 1998). Most of the mutations have been mapped to the 27 codons located at the center of rpo b gene that is known to bear mutations that confer rifampicin resistance in Escherichia coli. Many of the reported mutations are missense with a few cases of insertions and deletions also (Telenti et al. 1993a, b). Resistance to rifampicin is a relatively rare event and leads to selection of mutants that are already resistant to other components of short-course chemotherapy. Therefore, rifampicin resistance is often regarded as an excellent surrogate marker for MDR-TB (CDC 1993).

Isoniazid acts as the prodrug which is converted to an active form (isonicotinic acid or aldehyde- 
bearing groups or free radicals) by the kat G-encoded catalase-peroxidase enzyme in $M$. tuberculosis. Isoniazid resistance is due to conversion of Arg463 to Leu in the kat G protein (Heym et al. 1993, 1994). The second mechanism, conferring low level resistance, is mutation in the inh $A$ gene which encodes fatty acid synthase. This enzyme requires NADH as a cofactor; the mutant enzyme has been shown to have a lower affinity for NADH and cannot be saturated at NADH concentrations existing within $M$. tuberculosis (Johnsson \& Schultz 1994).

The target of fluoroquinolones action is the DNA gyrase, an ATP-dependent type II DNA topoisomerase that catalyses the negative supercoiling of DNA. This enzyme is made up of four units ( $22 \mathrm{~b} 2)$, which are encoded by the gyrA and gyr B genes respectively. Fluoroquinolones bind to the gyrase and inhibit the supercoiling of DNA. The gyrA and gyrB genes of $M$. tuberculosis have been cloned and mutations in the quinolone-binding site have been mapped (Takiff et al. 1994).

The present study represents the first report of molecular genetic analysis of clinical isolates of MDR M. tuberculosis from India. While association of rifampicin resistance with MDR is evident, we also demonstrate the utility of polymerase chain reaction-single strand conformational polymorphism (PCR-SSCP) for rapidly scoring mutations within the rpo b locus. Sequence analysis of the rpo b and gyrA loci shows that the more common missense mutations are also prevalent in the Indian isolates. This study reaffirms the growing incidence of MDR-TB in India.

\section{MATERIALS AND METHODS}

Clinical isolates of $M$. tuberculosis were procured from TB patients attending the out-patient departments at the All India Institute of Medical Sciences and the New Delhi Tuberculosis Center, New Delhi, India. Susceptibility testing of all the M. tuberculosis isolates was done by Bactec 460TB system. Minimum inhibitory concentration (MIC) was defined as the lowest drug concentration that inhibited bacterial growth by at least $99 \%$.

DNA was extracted from clinical isolates grown on LJ slants. The colonies were scraped, suspended in TE and subjected to freeze thawing (30 $\mathrm{min}$ at $70^{\circ} \mathrm{C}$ to $100^{\circ} \mathrm{C}$ for $10 \mathrm{~min}$ ). This was followed by treatment with lysozyme $(40 \mathrm{mg} / \mathrm{ml})$, SDS $(0.5 \%)$ and proteinase $\mathrm{K}(50 \mathrm{mg} / \mathrm{ml})$ at $37 \mathrm{C}$ for $2 \mathrm{hr}$. The protein and other contaminants were removed by CTAB precipitation. DNA was finally precipitated with 0.6 volumes of isopropanol. The precipitate was washed twice with 70\% ethanol, air dried and re-dissolved in water.
PCR was performed using 150 pmoles of each primer with $1 \mathrm{U}$ of $\mathrm{Taq}$ polymerase (Bangalore Genei, India), 200 mmoles of each dNTPs and 1.5 $\mathrm{mM}$ magnesium chloride. Mutations in the rpo b gene, conferring rifampicin resistance were detected with previously reported forward (5 TAC GGT CGG CGA GCT GAT CC 3) and reverse (5 TAC GGC GTT TCG ATG ATG AAC 3) primers. $250 \mathrm{mg}$ of template was amplified in 30 cycles in Perkin Elmer Cetus thermal cycler using the following conditions: $94^{\circ} \mathrm{C}-1 \mathrm{~min} ; 56^{\circ} \mathrm{C}-1 \mathrm{~min} ; 72^{\circ} \mathrm{C}$ $2 \mathrm{~min}$. The 350 bp rpo b amplicon was checked by electrophoresis on a $1.5 \%$ agarose gel.

Similar conditions were used for amplification of the kat $G$ genes mutational hot spot region using forward (5 GCC CGA GCA ACA CCC 3) and reverse (5 ATG TCC CGC GTC AGG 3) primers.

Sixteen isolates resistant to ofloxacin were checked for mutations in gyr $A$. For gyr $A$ amplification the primers used were 5 CAG CTA CAT CGA CTA TGC GA 3 and 5 GGG CTT CGG TGT ACC TCA T 3. The PCR amplification conditions were similar to that of rpo b gene amplification except that the annealing temperature was $45^{\circ} \mathrm{C}$. The amplicons were purified using Qiaquick PCR purification kit (Qiagen, USA). Samples were initially analyzed for SSCP. Briefly, the samples were heat denatured and electrophoresed on a composite gel $(0.25 \%$ agarose, $5 \%$ acrylamide and $5 \%$ glycerol). The gel was then silver stained (BioRad Silver stain Kit) and the DNA bands visualized. A change in the banding pattern as compared to the wild type $\mathrm{H} 37 \mathrm{Rv}$ strain was taken as indicative of mutation(s).

Cycle sequencing was performed following the manufacturers protocol using the cyclist $P f u$ Exokit (Stratagene, USA).The forward primers of $r p o$ $\mathrm{b}$ and gyr $A$ were used to sequence the SSCP positive samples. The $81 \mathrm{bp}$ and $30 \mathrm{bp}$ sequence corresponding to the hot spot region of the rpo b and gyr $A$ respectively, were read and compared with the respective sequences of standard H37Rv strain.

\section{RESULTS}

The MDR data for the 116 clinical isolates is shown in Table I. A majority of the strains (69\%) turned out to be resistant to at least one drug. It is important to mention at the outset that this study has a sampling bias in terms of the drug resistance cases and does not represent the observed frequency of the occurence of drug resistance $(\sim 13 \%)$ in Indian clinical isolates of M. tuberculosis (Ramalingaswami 1998). Between various drugs used in short term chemotherapy for tuberculosis, the isoniazid resistant was most common (56\%), 
TABLE I

Summary of multi drug resitance in Mycobacterium tuberculosis isolates from Indian patients

\begin{tabular}{lclc}
\hline Drugs & No. of isolates & MRD status & Samples \\
\hline Isoniazid & 65 & 1 drug & 15 \\
Rifampicin & 62 & 2 drugs & 25 \\
Ethambutol & 27 & 3 drugs & 20 \\
Streptomycin & 25 & 4 drugs & 5 \\
Ofloxacin & 16 & 5 drugs & 13 \\
None & 38 & & \\
\hline
\end{tabular}

Total No. of samples: 116

closely followed by rifampicin (53\%). The number of isolates resistance to the rest of the drugs was lesser (about $25 \%$ for ethambutol and streptomycin) and was least in case of fluoroquinolones. This data corelates well with the treatment regime followed in the TB clinics in India where fluoroquinolones represent the last line of drugs (Pande 1998). We observed that 58 isolates out of 62 positive for rifampicin resistance, were also resistant to atleast one other drug. Therefore, a majority $(93 \%)$ of the rifampicin resistant strains showed an association with resistance to other drugs thereby supporting earlier observations (Kapur et al. 1994, Williams et al. 1994, Musser 1995, Hasnain et al. 1998) on rifampicin as a surrogate marker for multidrug resistance.

The principle of PCR-SSCP is based on the fact that the two denatured strands of DNA (in this case PCR-amplified) adopt stable intramolecular conformations which may differ from the wild type upon mutation. This causes a change in the electrophoretic mobility of the strands. We utilized SSCP to conduct a primary screening of the rpo b, kat $G$ and gyr $A$ amplicons for the presence of mutations. The results of SSCP analysis reveal that while most of the rifampicin resistant strains did exhibit the expected mobility shifts correlating with point mutations, a very large percentage of isolates resistant to fluoroquinolone and carrying point mutations (revealed upon sequencing), however did not display altered electrophoretic mobility. The results of SSCP analysis of rpo b amplicons for few isolates are summarized in Table II. Some typical SSCP gel electrophoresis patterns corresponding to the rpo b (Fig. 1a), kat G (Fig. 1b) or gyr A amplicons (Fig. 1c) are represented.

The precise mutations within the drug resistant loci were identified by direct sequencing of the amplified regions. Sequence analysis (Fig. 2) of the rpo b gene hotspot for the rifampicin resistant isolates revealed the presence of many of the common mutations reported earlier ( Kapur et al. 1994) in addition to several novel mutations (data not

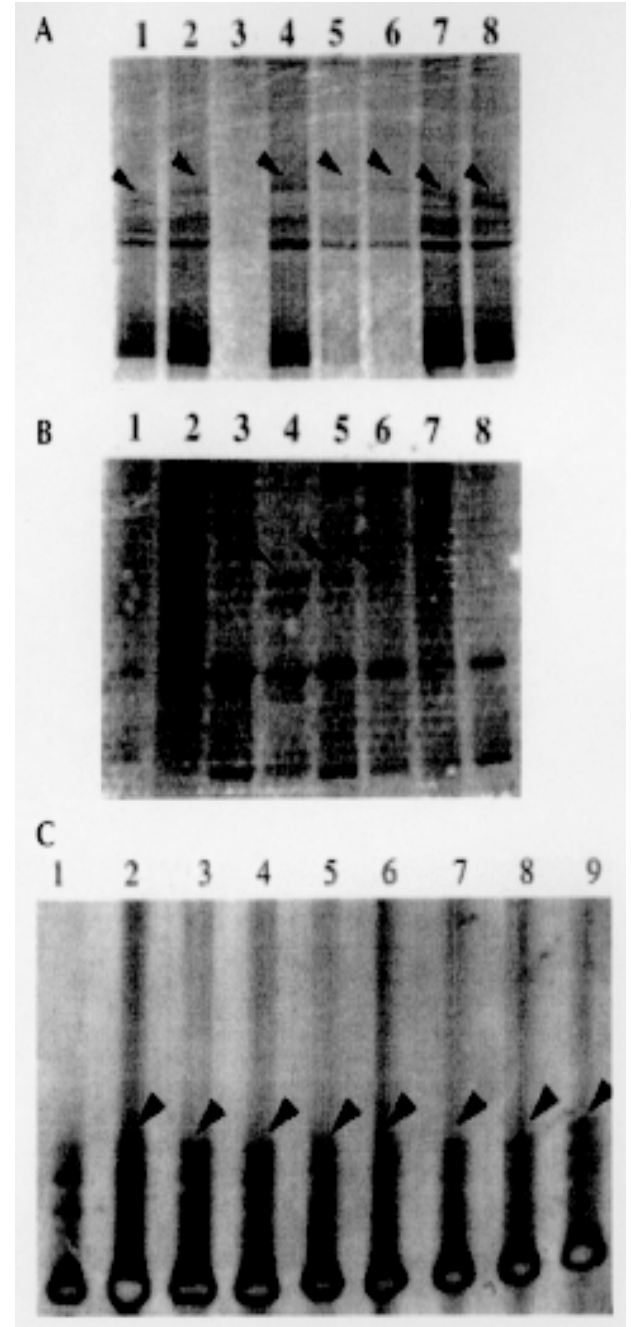

Fig. 1a: PCR-SSCP analysis of representative rpo b amplicons.gel picture of few rpo b amplicons. Arrow denotes the shift in bands due to the conformational polymorphism as a result of point mutation. Lanes 3 is the control lane; Fig $1 \mathrm{~b}$ : typical PCR-SSCP pattern displayed by kat G amplicons. Arrowmark denotes the DNA mobility shift due to the conformational polymorphism as a result of point mutation within the kat G locus; Fig. 1c: typical PCR-SSCP gel picture of few mutant gyr A amplicons. Arrow mark denotes the DNA mobility shift due to the conformational polymorphism as a result of point mutation within the gyr $A$ locus. Lanes 2 to 8 are amplicons from quinolone resistant strains while lane 1 has the wild type H37Rv strain.

shown). Mutations in the gyr $A$ gene, however were only of the types already reported earlier (Takiff et al. 1994 ). The S95T and A90V mutations were the two more common mutations within the Indian isolates of quinolone resistant $M$. tuberculosis. The amino acid changes caused by the corresponding point mutations in the DNA within the gyr A loci are shown in Fig. 3. 
TABLE II

Susceptibility to rifampicin and polymerase chain reaction-single strand conformational polymophism

(PCR-SSCP) results. '?' mark denotes that these isolates were resistant but were not positive in SSCP analysis

\begin{tabular}{|c|c|c|}
\hline $\begin{array}{l}\text { Isolate no. } \\
\text { analysis }\end{array}$ & $\begin{array}{l}\text { Rifampicin } \\
\text { sensitivity }(>1 \mathrm{mg} / \mathrm{ml})\end{array}$ & SSCP \\
\hline $\mathrm{C} 46$ & Sensitive & Negative \\
\hline C49 & Resistant & Positive \\
\hline C56 & Resistant & Positive \\
\hline C64 & Resistant & Positive \\
\hline C68 & Sensitive & Negative \\
\hline C71 & Resistant & Negative? \\
\hline C74 & Sensitive & Negative \\
\hline $\mathrm{C} 78$ & Resistant & Positive \\
\hline C80 & Sensitive & Negative \\
\hline C93 & Sensitive & Negative \\
\hline C97 & Sensitive & Negative \\
\hline A1 & Sensitive & Negative \\
\hline $\mathrm{A} 2$ & Sensitive & Negative \\
\hline A3 & Sensitive & Negative \\
\hline A4 & Sensitive & Negative \\
\hline A5 & Sensitive & Negative \\
\hline A6 & Sensitive & Negative \\
\hline A7 & Sensitive & Negative \\
\hline A8 & Sensitive & Negative \\
\hline A9 & Sensitive & Negative \\
\hline B1 & Sensitive & Negative \\
\hline B2 & Sensitive & Negative \\
\hline B3 & Sensitive & Negative \\
\hline B4 & Sensitive & Negative \\
\hline B5 & Sensitive & Negative \\
\hline B6 & Resistant & Positive \\
\hline B7 & Sensitive & Negative \\
\hline $\mathrm{F} 1$ & Resistant & Positive \\
\hline $\mathrm{F} 2$ & Resistant & Positive \\
\hline F3 & Resistant & Positive \\
\hline $\mathrm{F} 4$ & Resistant & Positive \\
\hline F5 & Resistant & Positive \\
\hline F6 & Resistant & Positive \\
\hline F7 & Resistant & Positive \\
\hline F8 & Resistant & Positive \\
\hline F9 & Resistant & Positive \\
\hline D1 & Sensitive & Negative \\
\hline D2 & Resistant & Negative? \\
\hline D3 & Resistant & Negative? \\
\hline D4 & Resistant & Negative? \\
\hline D6 & Resistant & Negative? \\
\hline D7 & Resistant & Positive \\
\hline D9 & Resistant & Positive \\
\hline D10 & Resistant & Positive \\
\hline D11 & Resistant & Positive \\
\hline D12 & Resistant & Positive \\
\hline D18 & Sensitive & Negative \\
\hline V1 & Resistant & Positive \\
\hline $\mathrm{V} 2$ & Resistant & Positive \\
\hline V3 & Resistant & Positive \\
\hline V4 & Resistant & Positive \\
\hline V5 & Resistant & Positive \\
\hline V6 & Resistant & Positive \\
\hline
\end{tabular}

\begin{tabular}{lll} 
V7 & Sensitive & Negative \\
V8 & Resistant & Positive \\
V9 & Resistant & Positive \\
V10 & Resistant & Positive \\
V11 & Resistant & Positive \\
V12 & Sensitive & Negative \\
V13 & Resistant & Positive \\
V14 & Resistant & Positive \\
V15 & Resistant & Positive \\
V16 & Resistant & Positive \\
V17 & Resistant & Positive \\
V18 & Resistant & Positive \\
\hline
\end{tabular}

\section{DISCUSSION}

In this study we present molecular genetic analysis of rifampicin, isoniazid and quinolone resistance in Indian clinical isolates of MDR $M$. tuberculosis. Point mutations within rpo b, kat $G$ and gyr $A$ genes respectively lead to amino acid polymorphism in the target protein of the drug resulting in drug resistance (Rattan et al. 1998). MDR does not appear to arise due to the acquisition of a transposable element or a plasmid carrying drug resistant marker, but is perhaps a reflection of stepwise acquisition of new mutations in the genes for different drug targets. Alterations in the chromosomal genes are random but get selected due to poor compliance or prescription. Inadequate prescription of chemotherapy, poor compliance of the drug regime and in recent times infection with HIV have caused an increase in the selection of MDR strains of M. tuberculosis (Lederberg 1998). A lack of monitoring programs and poor follow up of the patients health has caused an increase in relapse cases for TB. In most cases, the secondary infection is by drug resistant mycobacterium. What is of graver concern is the increasing incidence of primary infection by MDR-Mycobacterium tuberculosis (Hasnain et al. unpub. data). Patients infected with a rifampicin resistant strain of $M$. tuberculosis generally have a poor prognosis, particularly because rifampicin resistance is often associated with resistance to other frontline drugs.

We found a majority of the rifampicin resistant Indian isolates to be resistant to at least one another anti-tubercular drug, supporting the idea of using rifampicin resistance as a surrogate marker for MDR TB. PCR-SSCP analysis while offering a rapid method for detecting MDR particularly for rifampicin resistance however, has limitations as it failed to detect mutations within the gyr $A$ locus. These results representing a first report on the molecular genetic analysis of MDR in clinical isolates of $M$. tuberculosis from India have important bearing on the management and control of this reemerging infectious disease. 


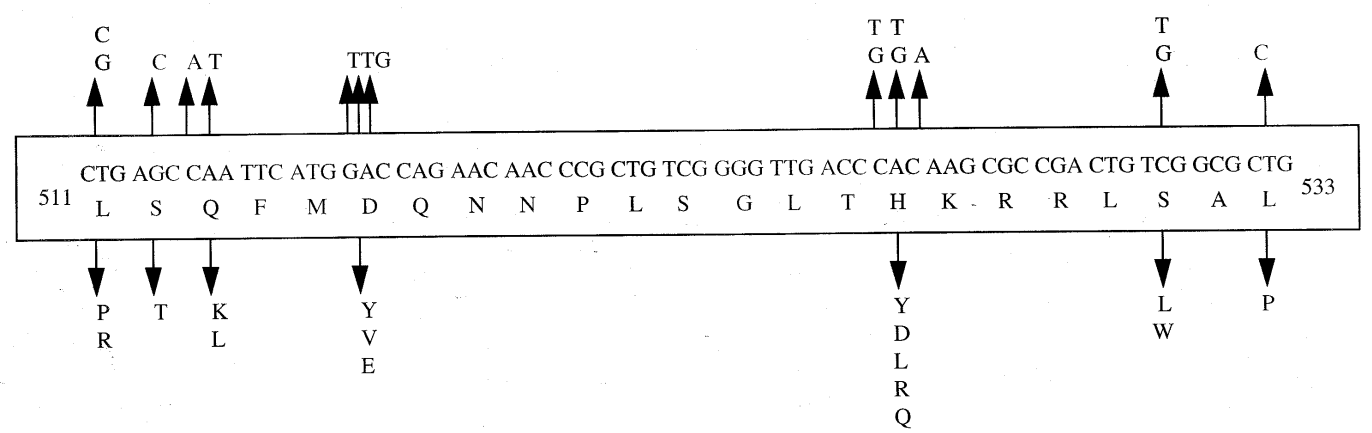

Fig. 2: common mutations in the rpo b gene. Top panel shows the nucleotide changes that are similar to previously reported mutations. The bottom panel depicts the corresponding amino acid changes within the RNA polymerase $b$ subunit.

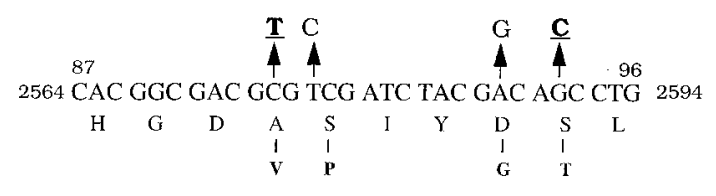

Fig. 3: mutations within the gyr A gene. The top panel shows the nucleotide change while the bottom panel depicts the corresponding amino acid changes. The common mutations in the Indian isolates are depicted in bold letters.

\section{ACKNOWLEDGMENTS}

To Mr Sunder Singh Bisht for his help in sequencing and SSCP.

\section{REFERENCES}

Bloom BR, Murray JL 1992. Tuberculosis: commentary on a reemergent killer. Science 257: 1055-1064.

CDC - Centers for Disease Control 1993. Initial therapy for tuberculosis in the era of multi-drug resistance. recommendations of the advisory council for the elimination of tuberculosis. MMWR 42: RR-7.

Hasnain SE, Amin A, Siddiqi N, Shamim M, Jain NK, Rattan A, Katoch VM, Sharma SK 1998. Molecular genetics of multiple drug resistance (MDR) in $M y$ cobacterium tuberculosis, p. 35-40. In RLSinghal \& OP Sood (eds), Drug Resistance: Mechanism and Management, Proceedings of the Fourth Annual Ranbaxy Science Foundation Symposium Communicore Publishers, New Delhi, India

Heym B, Alzari PM, Honore N, Cole ST 1994. Missense mutations in the catalase-peroxidase gene, $\mathrm{katG}$, are associated with isoniazid resistance in Mycobacterium tuberculosis. Mol Microbiol 15: 235245.

Heym B, Zhang Y, Poulet S, Young D, Cole ST 1993. Characterization of the katG gene encoding a catalase-peroxidase required for the isoniazid susceptibility of Mycobacterium tuberculosis. J Bacteriol 175: 4255-4259.

Honore N, Cole ST 1993. The molecular basis of rifampin resistance in Mycobacterium leprae. Antimicrob Agents Chemother 37: 414-418.
Iseman MD 1994. Evolution of drug-resistant tuberculosis: A tale of two species. Proc Natl Acad Sci USA 91: 2428-2429.

Johnsson K, Schultz PG 1994. Mechanistic studies of the oxidation of isoniazid by the catalase peroxidase from Mycobacterium tuberculosis. J Am Chem Soc 116: 7425-7426.

Kapur V, Ling Ling LI, Iordanescue S, Krieswirth BN, Musser JM 1994. Characterization by automated DNA sequencing of mutations in the gene (rpo b) encoding the RNA polymerase $\mathrm{b}$ subunit in rifampin Mycobacterium tuberculosis strains from New York and Texas. J Clin Microbiol 32: 1095-1098.

Lederberg J 1998. The future of infectious disease, p.514. In RLSinghal \& OP Sood (eds), Drug Resistance: Mechanism and Management, Proceedings of the Fourth Annual Ranbaxy Science Foundation Symposium Communicore Publishers, New Delhi, India.

Miller LP, Crawford JT, Shinnick TM 1994. The rpo b gene of Mycobacterium tuberculosis. Antimicrob Agents Chemother 38: 805-811.

Musser JM 1995. Antimicrobial agent resistance in mycobacteria: molecular genetic insights. Clin Microbiol Rev 8: 496-514.

Pande JN 1998. Multidrug resistant tuberculosis: Current concepts and future directions for management, p. 87-90. In RLSinghal \& OP Sood (eds), Drug Resistance: Mechanism and Management, Proceedings of the Fourth Annual Ranbaxy Science Foundation Symposium Communicore Publishers, New Delhi, India.

Ramalingaswami V 1998. Opening address, p. 1-3. In RLSinghal \& OP Sood (eds), Drug Resistance: Mechanism and Management, Proceedings of the Fourth Annual Ranbaxy Science Foundation Symposium Communicore Publishers, New Delhi, India.

Rattan A, Kalia A, Ahmad N 1998. Multidrug resistant tuberculosis: Molecular perspectives. Emerg Infec Dis 4: 195-209.

Takiff HE, Salazar L, Guerrero C, Philipp W, Huang WM, Kreiswirth B, Cole ST, Jacobs Jr WR, Telenti A 1994. Cloning and nucleotide sequence of the Mycobacterium tuberculosis gyrA and gyr B genes, and characterization of quinolone resistance mutations. Antimicrob Agents Chemother 38: 773-780. 
Telenti A, Imboden P, Marchesi F, Lowrie D, Cole S, Bodmer T 1993a. Detection of rifampicin-resistance mutations in M. tuberculosis. Lancet 341: 647650.

Telenti A, Imboden P, Marchesi F, Schmidheini T, Bodmer T 1993b. Direct automated detection of rifampicin-resistant Mycobacterium tuberculosis by polymerase chain reaction and single-strand conformation polymorphism analysis. Antimicrob Agents Chemother 37: 2054-2058.

Williams DL, Wanguespack C, Eisenach KD, Bates JH, Crawford JT 1994. Characterization of rifampin resistance in pathogenic mycobacteria. Antimicrob Agents Chemother 38: 2380-2386. 